\title{
Glaucoma Detection from Retinal Images
}

\author{
Vishnubhotla Poornasree1, Vijayagiri Ashritha1, Venumula Deeksha Reddy¹, J. Srilatha² \\ ${ }^{1}$ UG Scholar, ${ }^{2}$ Assistant Professor \\ 1,2Department of Computer Science and Engineering, \\ 1,2Stanley College of Engineering and Technology for Women, Hyderabad, Telangana, India
}

\begin{abstract}
How to cite this paper: Vishnubhotla Poornasree | Vijayagiri Ashritha | Venumula Deeksha Reddy | J. Srilatha "Glaucoma Detection from Retinal Images" Published in International Journal of Trend in Scientific Research and Development (ijtsrd), ISSN: 24566470, Volume-3 | Issue-4, June 2019, pp.437-439, URL: https://www.ijtsrd.c om/papers/ijtsrd23 732.pdf

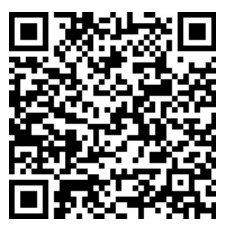
IITSRD23732

Copyright (c) 2019 by author(s) and International Journal of Trend in Scientific Research and Development Journal. This is an Open Access article distributed under the terms of the Creative Commons Attribution License (CC BY 4.0) (http://creativecommons.org/licenses/ by $/ 4.0$ )

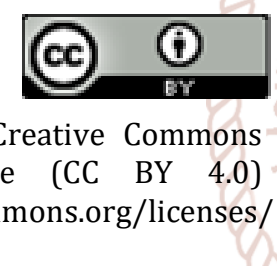

\section{INTRODUCTION}

A human eye is secreted by a watery fluid called aqueous humor. This fluid causes an intra-ocular pressure (IOP) on the inner surface of the eye. The pressure limits up to 20 mmHg. The IOP is kept constant by an even distribution of fluids that flow inside an eye. The fluids are the aqueous humor produced in the eye and the fluid that leaves a human eye by an eye's drainage system. The disturbance in the distribution of fluid inside an eye leads to change in the intra-ocular pressure. This change in the pressure inside an eye damages the optic nerve head that internally changes the location of the optic cup and optic disc. The case in which the IOP increases and crosses a limit of $30 \mathrm{mmHg}$, reduces the thickness of the retinal nerve fiber layer (RNFL) that damages the optic nerve consequently. This disturbs the interconnection between the photoreceptors. Photoreceptors of a human eye are useful in the transmission of the visual information to the brain. On a severe attack of glaucoma, the photoreceptors don't work in the visual field.

In glaucoma affected eye, the optic cup size starts increasing gradually that whacks the neuro-retinal rim (NRR) as it is placed between the surfaces of the optic cup and the optic disc. According to the ISNT quadrants, a normal eye has its cup to disc ratio (CDR) as 0.5 . As a result of an increase in cup size, the CDR value also gets affected and will be greater

than 0.5 , which indicates glaucoma. It is a challenging case for a person to detect glaucoma at its earliest stage as it shows an imperceptive growth that ultimately leadsto complete and irreversible blindness. Glaucoma is commonly observed in people with an age over and above 60 years. While the other age group can be affected based on the family background and their genetic behaviour. Early detection of glaucoma can be done by the fundus images of an eye.

Fundus photographs are obtained by capturing a photograph of the fundus. Specialized fundus camera consists of an intricate microscope that is used in fundus photography. The main structures that can be visualized on a fundus photo are the retina, optic disc and macula. Fundus photography can be performed with coloured filters that are red, blue and green colours, commonly called as RGB.

\section{Problem Definition}

The aim is to design a computer-aided glaucoma detection system that detects glaucoma at an early stage from the fundus images of the human eye. The implementation is based on CNN.

\section{RELATED WORKS}


Glaucoma is one of the major eye diseases that lead to irreversible loss of vision. This damage cannot be easily detected as glaucoma does not show any symptoms in its early stage. An automated computer-aided system will help ophthalmologists in early detection of glaucoma. The existing approaches involve the extraction of the optic cup and optic disc followed by elicitation of the properties such as cup to disc ratio and ISNT ratio to classify glaucomatous and healthy images.

The detailed reviews of the existing work on computer-aided diagnosis for glaucoma using digital fundus image are observed that most of the algorithms follow the two-stage structure, they are feature extraction and classification. The Wavelet, Gabor transform, Higher order spectra (HOS), etc., are the most used techniques for feature extraction. In classification, artificial neural network (ANN), supportvector machine (SVM), K-nearest neighbour (KNN) predict glaucoma. The design of such hand-crafted features is a tedious job and time-consuming. These features are strongly related to expert knowledge and having restricted representation power. It cannot show the discriminative power for a huge dataset. Deep learning overcomes the problem and enhances the classification. SVM classifier achieved maximum performance of 97\% accuracy for nineteen features using 1000 fundus images. Convolutional neural networks for glaucoma detection is based on detecting the region of interest (ROI) from segmented images. The proposed system is fully automated deep learning architecture which can classify even early stage of glaucoma. Convolution neural networks (CNN) extract the localized features from input images and convolution is performed with image patches using filters. Filter responses are pooled repeatedly and re-filtered, and the output feature vector of resulting images on deep feed-forward network architecture are eventually classified. The details of the developed model are presented in the subsequent sections.

\section{PROPOSED SYSTEM}

Glaucoma detection from retinal images is implemented based on CNN. CNN is the most efficient method used in medical analysis and an effective technique to detect the region of interest and also image classification.

\section{Module Description}

The three modules of the project are:

1. Image Pre-processing module

2. Feature Extraction module

3. Classification module

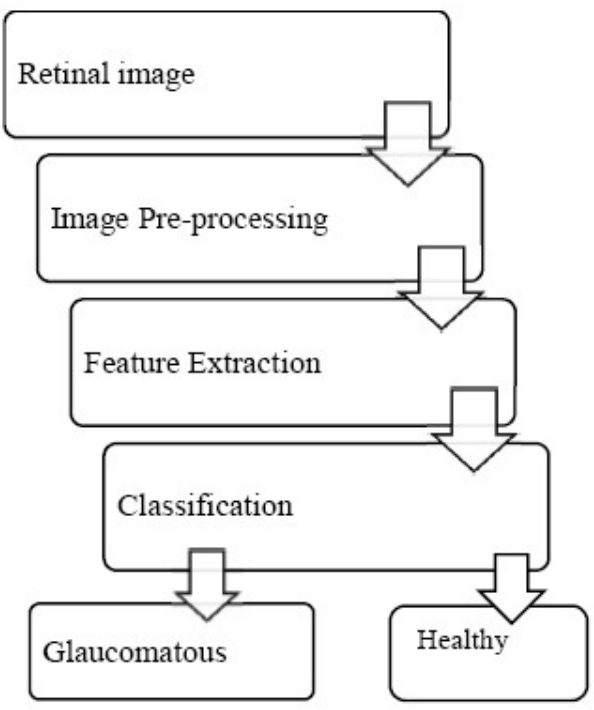

\section{Image Pre-processing}

Pre-processing defines the operations on images. The input and output are intensity images. These images are of the same kind as the original data capture. The intensity image is usually represented by a matrix of image function values such as brightness, contrast. Pre-processing improves the image data that downscales the unwanted distortions and enhances the image features that facilitates further processing. The removal of noise from the image, reshaping and resizing of an image can be considered to be a part of the pre-processing phase.

\section{Feature Extraction}

This is the most crucial phase of glaucoma detection. Feature extraction is a type of reducing the dimensions that efficiently represents the region of interest from an entire image vector. This approach is useful when image sizes are large and the feature representation is required to perform the image matching tasks and information retrieval. Detection of Region of Interest (ROI) comes under the feature extraction phase.

In the project, the optic cup and optic disc form of the region of interest. The value of the cup to disc ratio is acquired from the ROI.

\section{Classification}

Image classification is the task of classifying the data into multiple classes from a spatial raster image. Image classification is done upon analyzing the properties of various features from the images and organizes this data into different categories or classes.

The fundus image taken is classified into two categories: healthy eye, glaucomatous eye. 


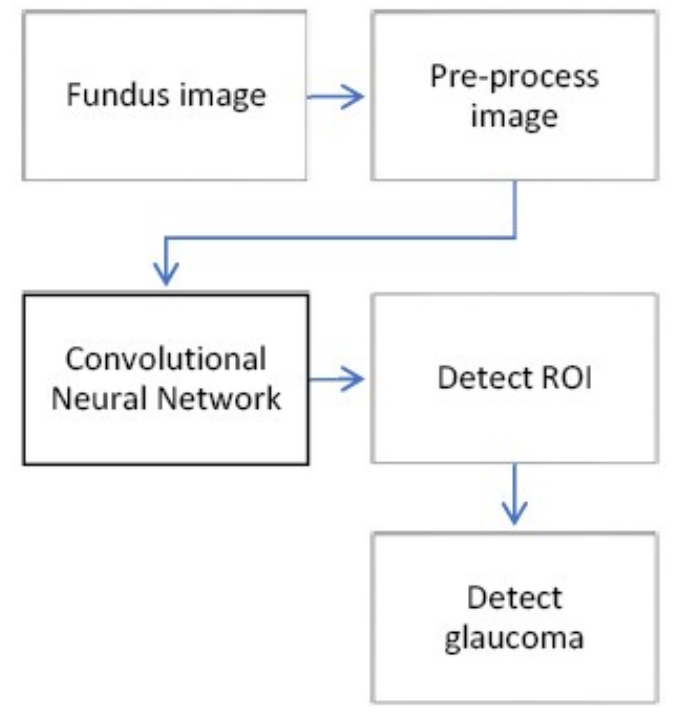

\section{Convolutional Neural Network}

A convolutional neural network follows a traditional neural network approach. CNN consists of an input, multiple hidden layers and an output layer. The hidden layers of CNN are typical in nature and consists of multiple functional layers. The convolutional layers, pooling layers, fully connected layers and normalization layers are the most used functional layers in the hidden layers of CNN.

\section{Convolutional layer}

The convolutional layer can be said to be the core building block of a CNN. Each convolutional neural network

starts with this convolutional layer. The parameters to this layer consists of a set of learnable filters or kernels. These kernels have a receptive field that can accept the input volume. During the forward pass, each filter is convolved across the dimensions of the input volume, computing a dot product between the entries of the filter and the input. As a result, the network is trained over the filters that activate when it detects the feature at some spatial position in the input.

\section{Pooling layer}

Convolutional networks also include local and global pooling layers. Pooling layers generally reduce the dimensions of the data. The reduced dimension is obtained by combining the outputs of neuron clusters into a single neuron. These newly formed clusters are fed forward to the next layers over the network. Local pooling combines small clusters typically of matrix size, $2 \times 2$. While global pooling acts on all the neurons of the convolutional layer. Pooling computes the max or average value from a cluster of neurons. Max pooling uses the maximum value at the prior layer. Average pooling uses the average value at the prior layer.

\section{Fully connected layer}

The high-level reasoning in a neural network is done through fully connected layers. Neurons in a fully connected layer are connected to all activations in the previous layer, as in a non-convolutional artificial neural network. The activations can be computed with matrix multiplication followed by an offset. Fully connected layers connect each neuron in one layer to each neuron in another layer.

\section{Activation functions}

Activation functions introduce non - linear properties to the network. They decide if a neuron in the network has to be activated or

not by calculating the weighted sum. The activation functions used in the project are:

\section{Rectified Linear Unit}

Applying ReLU function to the feature maps to increase the non - linearity in the network. It effectively removes the negative values from an activation map by setting them to zero.

$$
g(x)=x^{+}=\max (0, x)
$$

\section{Sigmoid Function}

A sigmoid function is a non-negative derivative, bounded, differentiable, a real function that is defined for all real input values at each point. The sigmoid function is a characteristic and a smooth continuous function. The outputs of the function range between -1 and 1 .

$$
f(x)=\frac{1}{1+e^{-g(x)}}
$$

\section{CONCLUSION}

The proposed computer-aided system will be a helpful way for the early detection of glaucoma. The classification of the fundus images as glaucomatous and healthy is based upon the region of interest obtain ed after passing the image over the convolutional neural network. The glaucomatous images are classified and displayed in an excel sheet.

The future work may involve extracting more parameters from the fundus image and also predicting the stage at which the eye is affected by glaucoma.

\section{REFERENCES}

[1] Gayathri Devi T.M.,Sudha S, Suraj P, "Glaucoma Detection from Retinal Images", Ieee Sponsored 2nd International Conference on Electronics and Communication Systems (ICECS 2015).

[2] Jose Ignacio Orlando, Elena Prokofyeva, Mariana Del Fresno, Mathew B. Blaschko, "Convolutional Neural Network Transfer for Automated Glaucoma Identification", 26 January 2017.

[3] 1nacer Eddine Benzebouchi, 2nabiha Azizi, 3seif Eddine Bouziane, "Glaucoma Diagnosis Using Cooperative Convolutional Neural Networks", (Jan 2018).

[4] Boosting Convolutional Filters with Entropy Sampling for Optic Cup and Disc Image Segmentation from Fundus Images Julian G. Zilly, Joachim M. Buhmann, and Dwarikanath Mahapatra, (2015).

[5] Aquino, A. Gegundez-Arias, M. Marin., D. Detecting the optic disc boundary in digital fundus images using morphological edge detection and feature extraction techniques. IEEE Trans. Medical Image 2011.

[6] Haleem, M. S., Han, L., van Hemert, J., and Li, B., "Automatic extraction of retinal features from colour retinal images for glaucoma diagnosis: A review," Computerized Medical Imaging and Graphics 37(7), 581-596 (2013)

[7] Sermanet, P., Eigen, D., Zhang, X., Mathieu, M., Fergus, R., and LeCun, Y., "Overfeat: Integrated recognition, 
International Journal of Trend in Scientific Research and Development (IJTSRD) @ www.ijtsrd.com eISSN: 2456-6470

localization and detection using convolutional networks," in [International Conference on Learning Representations], (2014).

[8] Jaderberg, M., Vedaldi, A., and Zisserman, A., "Speeding up convolutional neural networks with low rank expansions," in [Proceedings of the British Machine Vision Conference], BMVA Press (2014).
[9] Chakravarty, A. and Sivaswamy, J., "Glaucoma classification with a fusion of segmentation and imagebased features," in [Biomedical Imaging (ISBI), 2016 IEEE 13th International Symposium on], 1, 689-692, IEEE (2016).

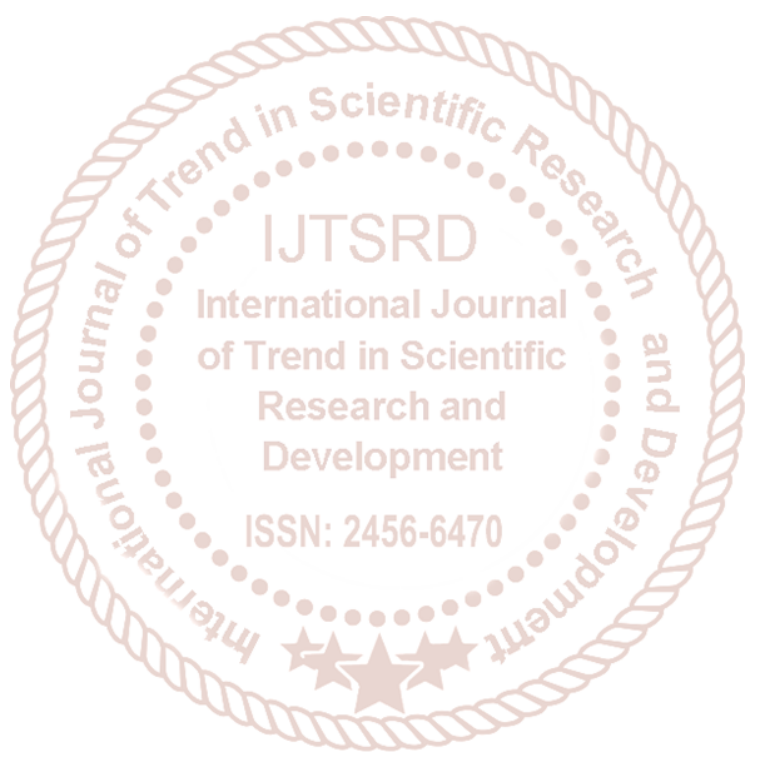

